\title{
Functional in silico analysis of human tyrosinase and OCAI associated mutations
}

\begin{abstract}
Oculocutaneous albinism type 1 (OCA1) is an autosomal recessive disorder caused by mutations in the tyrosinase gene. OCA1 exists in two forms: OCA1A and OCA1B. OCA1A is caused by a full loss of the human tyrosinase protein (Tyr), leading to an absence of pigment in skin, hair, and eyes, while OCA1B has reduced Tyr catalytic activity and pigment. The current understanding of the disease is hampered by the absence of information regarding the alterations of protein structure and the effects leading to either form of OCA1. Here, we used computational methods to find a general mechanism for establishing this link. Tyr and mutant variants were built through homology modeling, glycosylated in silico, minimized, and simulated using $100 \mathrm{~ns}$ molecular dynamics in water. For OCA1B mutants, cavity size is linked to $\Delta \Delta \mathrm{G}$ values for mutants, suggesting that partial loss of Tyr is associated with the destabilizing effect of the EGF-like domain movement. In OCA1A, active site mutation simulations indicate that the absence of $\mathrm{O}_{2}$ leads to protein instability. OCA1B mutants are described in severity by the size of the cavity within the EGF-Tyr interface, while active site OCA1A mutants are unable to fully coordinate copper, leading to an absence of $\mathrm{O}_{2}$ and Tyr instability. In patients with known genotypes, free energy changes may help identify the severity of the disease by assessing either the allosteric effect of the EGF-Tyr cavity in OCA1B or the active site instability in OCA1A.
\end{abstract}

Keywords: human tyrosinase, genetic mutations, oculocutaneous albinism 1, molecular modeling, classification of disease-causing mutations
Volume 9 Issue 3 - 2020

\section{Milan Patel,Yuri Sergeev}

National Eye Institute, National Institutes of Health, Bethesda, MD 20892, USA

\author{
Correspondence: Yuri Sergeev, National Eye Institute, \\ National Institutes of Health, Bethesda, MD 20892, USA, \\ Tel +(30I) 594 7053, Fax +(496) 402 I2I 4, \\ Email sergeevy@nei.nih.gov
}

Received: August II, 2019 | Published: August 24, 2020
Abbreviations: Tyr, tyrosinase; OCA1, oculocutaneous albinism 1; OCA1A, oculocutaneous albinism 1 type A; OCA1B, oculocutaneous albinism type B; RMSD, root-mean-square deviation; EGF, epidermal growth factor; EGF-Tyr cavity, a cavity in the interface between tyrosinase and EGF-like domains; MD, molecular dynamics; UMS, unfolding mutation screen

\section{Introduction}

Human tyrosinase is a type 1 trans-membrane and coppercontaining glycoenzyme that catalyzes the rate-limiting step of melanin pigment production in melanosomes. ${ }^{1,2}$ Mutations in the tyrosinase gene $(T Y R)$ can lead to oculocutaneous albinism Type 1 (OCA1), an autosomal recessive disease. Phenotypic signs of OCA1 include the absence (Type A) or reduction (Type B) of pigment in skin, hair, and eyes. OCA1A is associated with a complete loss of tyrosinase function, while OCA1B exhibits reduced tyrosinase enzymatic activity. ${ }^{3}$ Nearly 350 TYR mutations have been identified as causes of OCA1 in patients with established diagnosis, $77 \%$ of which are missense mutations (OCA1A $-67 \%$ and OCA1B $-33 \%)^{4}$

While recent attempts at crystallography of human tyrosinase have been unsuccessful, ${ }^{5}$ the crystal structure of human tyrosinaserelated protein 1 domain (residues 1-537) was determined at 2.3 $\AA$ resolution, and can be used as a reference for modeling intramelanosomal domains of mammalian tyrosinases due to their $40-50 \%$ sequence identity. ${ }^{6,7}$ The active site contains four $\alpha$-helices containing six His residues that coordinate two zinc ions involved in the catalytic mechanism of Tyrp1. These zinc ions are partially stabilized by one water molecule between them. Crystal structures of bacterial and fungal tyrosinase species are readily available; however, in crystallographic studies, the majority of protein structures did not demonstrate protein enzymatic activities, indicating possible differences from a protein native state. Also, a human tyrosinase structure is needed to answer OCA1 mutation-related questions.

Tyr is an N-linked glycoprotein that relies on glycosylation for its proper folding. ${ }^{8,9}$ Glycosylation stabilizes human Tyr during the process of translocation from the endoplasmic reticulum (ER) to the cytoplasm of the melanocyte. ${ }^{4}$ OCA1A mutants have been shown to destabilize the Tyr structure, resulting in a loss of enzymatic activity and proteolytic degradation. OCA1B also results in structural destabilization, but to a lesser degree, meaning the mutant appears to produce lower levels of melanin in melanocytes compared to the wild type..$^{4,10}$ The unfolding mutation screen (UMS) was developed in order to understand and evaluate the effect of missense mutations on protein folding and thermodynamic stability. ${ }^{11}$ This program uses protein unfolding curves and thermodynamic changes in Gibbs free energy $(\Delta \Delta \mathrm{G})$ to calculate propensities of mutations in global mutagenesis. The results are then projected onto a protein model to highlight the critical residues and regions of structural importance to the protein.

While the difference in phenotype between OCA1A and OCA1B have been identified, the difference in the molecular mechanism leading to these results have yet to be discovered. Here, we used computational methods to find a general mechanism for establishing a link between mutation changes at the Tyr protein level and OCA1 disease phenotypes. Tyr and mutant variants were built through homology modeling, glycosylated in silico, minimized, and simulated using $100 \mathrm{~ns}$ molecular dynamics in water. For OCA1B mutants, cavity size is linked to free energy changes for mutants, suggesting that partial loss of Tyr in OCA1B is associated with the destabilizing effect of the EGF-like domain movement. In OCA1A, active site mutation simulations demonstrated that the absence of $\mathrm{O}_{2}$ in an active 
site causes the inability to fully coordinate copper atoms and leads to Tyr protein instability. In patients with known phenotypes, $\Delta \Delta \mathrm{G}$ values produced in our work may help identify the severity of OCA1 by assessing either the allosteric effect of the EGF-Tyr cavity in OCA1B or the active site instability in OCA1A.

\section{Material and methods}

\section{Molecular modeling}

A human homology model of the intra-melanosomal domain of human tyrosinase (TyrD, residues 19-469) was built, and refined using 2 ns molecular dynamics (MD). Ion concentration was added as a mass fraction with $0.9 \% \mathrm{NaCl}$ used. Simulation temperature was set to $298 \mathrm{~K}$ with a water density of 0.997 at $\mathrm{pH} 7.4$. The cell size extended to $10 \AA$ beyond each side of the protein in the shape of a cube with dimensions $92.9 \AA$ x $92.9 \AA$ x $92.9 \AA$. Each mutation was run through molecular dynamics in YASARA using an AMBER03 forcefield, with a timestep of $5 \mathrm{fs}$. Tyr was run through the Unfolding Mutation Screen, and the atomic model was uploaded to the ocular proteome website (https://neicommons.nei.nih.gov/\#/proteome). ${ }^{11,12}$ Later, the structure of tyrosinase was glycosylated in-silico as follows. The OCA1B PDB files were glycosylated at five biologically likely Asn sites (N111, N161, N230, N337, and N371) using the online web tool Glycam-Web (http://glycam.org). The exact sugar composition in each N-glycosylation site is not known; the most biologically common eukaryotic core glycan was chosen and attached to the site: ( $\mathrm{N}$-acetylglucosamine, $\beta$-D-mannose, $\alpha$-D-mannose). ${ }^{13,14} \mathrm{Using}$ the Build Structure function in UCSF Chimera, ${ }^{15}$ bonds were added between N's $\mathrm{N} \delta 2$ atom and $\mathrm{N}$-acetylglucosamine's $\mathrm{C} 1$ atom. The $\mathrm{O} 1$ and $\mathrm{HO} 1$ atoms on $\mathrm{N}$-acetylglucosamine and $\mathrm{HD} 21$ atom on $\mathrm{N}$ were deleted.

\section{Mutant modeling}

Eleven OCA1A (H180N, H180R, H202R, H202Q, H211R, H363T, H363R, H363Y, H367R, H367Y, H390T) and seven OCA1B (W39R, R77G, K142M, S323R, T325A, M370V, V393D) mutations were generated using the Edit $>$ Swap $>$ Residue function on the Tyr PDB file in YASARA (http://www.yasara.org/). Tyrosinases are synthesized in the endoplasmic reticulum (ER) and function in melanosomes at two different $\mathrm{pH}$ conditions, $\mathrm{pH} 7.4$ and $\mathrm{pH}$ 5.0, respectively. ${ }^{16}$ Therefore, the mutations were simulated in a water box using the YASARA runfast.mcr macro, with OCA1A at $\mathrm{pH} 7.4$ (to replicate the ER environment) and OCA1B at $\mathrm{pH} 5.0$ (to replicate the melanosome environment). ${ }^{17,18}$ The simulation was run as described in the molecular modeling methods section. PDB files were created at 0 ns after minimization and at $100 \mathrm{~ns}$.

\section{Active site alterations}

Tyrosinase and tyrosinase-related proteins each have coordinating molecules within the active site. ${ }^{13}$ In order to define their role in tyrosinase function, the Tyr and OCA1A mutant structures were simulated without the dioxygen molecule. The dioxygen that binds copper within the active site were deleted from the PDB file. After deletion, each structure was energy-minimized in a water box using the AMBER14 force field, and subsequently run through molecular dynamics. The same parameters as the original mutants were used.

\section{Structure comparison}

Tyr and OCA1 mutant structures were compared in Chimera. ${ }^{15}$ Structures were overlaid onto one another and the RMSD was calculated using the MatchMaker function. The best-aligning pair of chains between reference and match structure was chosen for chain pairing. The Needleman-Wunsch algorithm using BLOSUM-62 was used for calculation. Matching was iterated by pruning long atom pairs until no pair exceeded 2.0 angstroms in order to remove far apart residues on the "match list" used to superimpose the structures.

\section{Accessible area and cavity analysis}

Tyr and all OCA1B mutant structure simulations were analyzed at 0 ns and $100 \mathrm{~ns}$ using MOLE 2.5 for cavities within the protein. Loading a protein structure leads to automatic detection of cavities based on an algorithm. ${ }^{19}$ The default parameters for this algorithm are $3.00 \AA$ for the probe radius, $1.25 \AA$ for the interior threshold, and $5.00 \AA$ for the minimum depth. These parameters were used in the calculation of cavities for the Tyr and OCA1B mutants. The three largest cavities of each structure were summed and used for analysis, assumed to be a reasonable approximation for the total cavity volume in each protein structure. For mutant variants, the effect of MD simulations was estimated as a difference between parameter changes in protein structure (mut) at $100 \mathrm{~ns}$ and $0 \mathrm{~ns}$ structure (wt). The change in solvent-accessible surface area (SA) over $100 \mathrm{~ns}$ between structures was described as $\Delta \Delta \mathrm{SA}=\Delta \mathrm{SA}_{\text {mut }}-\Delta \mathrm{SA}_{\mathrm{wt}}$. The change in solventaccessible volume $(\mathrm{V})$ over $100 \mathrm{~ns}$ between structures was described as $\Delta \Delta \mathrm{V}=\Delta \mathrm{V}_{\text {mut }}-\Delta \mathrm{V}_{\mathrm{wt}}$. The change in cavity volume $\left(\mathrm{V}_{\text {cav }}\right)$ over 100 ns between structures was described as $\Delta \Delta \mathrm{V}_{\text {cav }}=\Delta \mathrm{V}_{\text {cav_mut }}-\Delta \mathrm{V}_{\text {cav_wt }}$.

\section{Unfolding mutation screen}

Global mutagenesis was conducted on Tyr, and each mutant was characterized by a thermodynamic change in Gibbs free energy $(\Delta \Delta \mathrm{G})$. These values were calculated using the semi-empirical method (FoldX) and standardized on a $0-1$ scale, named the unfolding parameter, known as the fraction of protein in the unfolded state. ${ }^{11,20}$ This parameter described the predicted effect of each mutation as weak $(0-0.19)$, moderate $(0.2-0.8)$, or severe $(0.81-1)$. The Unfolding Mutation Screen (UMS) also outputted a foldability parameter to show critical residues in protein folding. This parameter is a sum of severity-weighted unfolding propensities for the 20 mutations generated at a specific residue. Residues with the highest foldability were considered critical for protein folding. ${ }^{11,14}$

\section{Statistical analysis}

Within the UMS pipeline, a procedure called "internal control" verifies the quality of the protein structure by mutating each residue from the protein sequence to itself. ${ }^{11}$ The $\Delta \Delta \mathrm{G}$ values of each identity mutation are calculated and then converted to unfolding propensities. The quality of the protein model is then determined by calculating the mean, standard deviation, p-value, and $95 \%$ confidence interval for those propensities. Each unfolding propensity should have a value of 0.5 . Statistical significance of OCA1 structural parameters were assessed using Pearson's correlation coefficient and an adjusted $\mathrm{R}$-squared value. The Pearson's correlation coefficient formula is:

$$
r=\frac{n\left(\sum x y\right)-\left(\sum x\right)\left(\sum^{y}\right)}{\sqrt{\left(n \sum^{2} x^{2}-\left(\sum^{x}\right)^{2}\right)\left(n \sum^{y^{2}}+\left(\sum^{y}\right)^{2}\right)}}
$$
where $\mathrm{n}$ is the number of points. The adjusted R-squared formula is:

$$
R_{a d j}^{2}=1-\left[\frac{\left(1-R^{2}\right)(n-1)}{n-k-1}\right],
$$
, where $\mathrm{n}$ is the number of points and $\mathrm{k}$ is the number of independent repressors. 


\section{Results}

\section{Homology model of Tyr}

Tyr homology model contains 12 alpha helices of at least one turn, four beta sheets, and seven disulfide bonds. The first five disulfide bonds are situated in the EGF-like domain, a repeat found in many extracellular and cell surface proteins (Figure 1A), (Figure 2). This domain is also found in homologous tyrosinases, Tyrp1 and Tyrp2. While the typical EGF-like repeat contains three disulfide bridges, the Tyr structure contains two more within the same region, providing more stability.

The active site of Tyr contains a bundle motif containing four $\alpha$-helices (H9, H10, H16, H19) surrounding a copper-dioxygencopper complex involved in the catalytic mechanism (Figure 1B), (Figure 3). ${ }^{1}$ Within the active site are six His residues that coordinate the two coppers, which are held together 2.7-2.8 $\AA$ apart. The first copper, $\mathrm{CuA}$, is coordinated by H180, H202, and H211 (Figure 3A). $\mathrm{H} 180$ is in $\alpha$-helix H9, H211 is located within $\alpha$-helix H10, and H202 is located in between $\alpha$-helix $\mathrm{H} 9$ and $\alpha$-helix H10. The second copper, $\mathrm{CuB}$, is coordinated by H363, H367, and H390 (Figure 3B). H363 and $\mathrm{H} 367$ are situated within $\alpha$-helix H16, and H390 is situated within $\alpha$-helix H19.

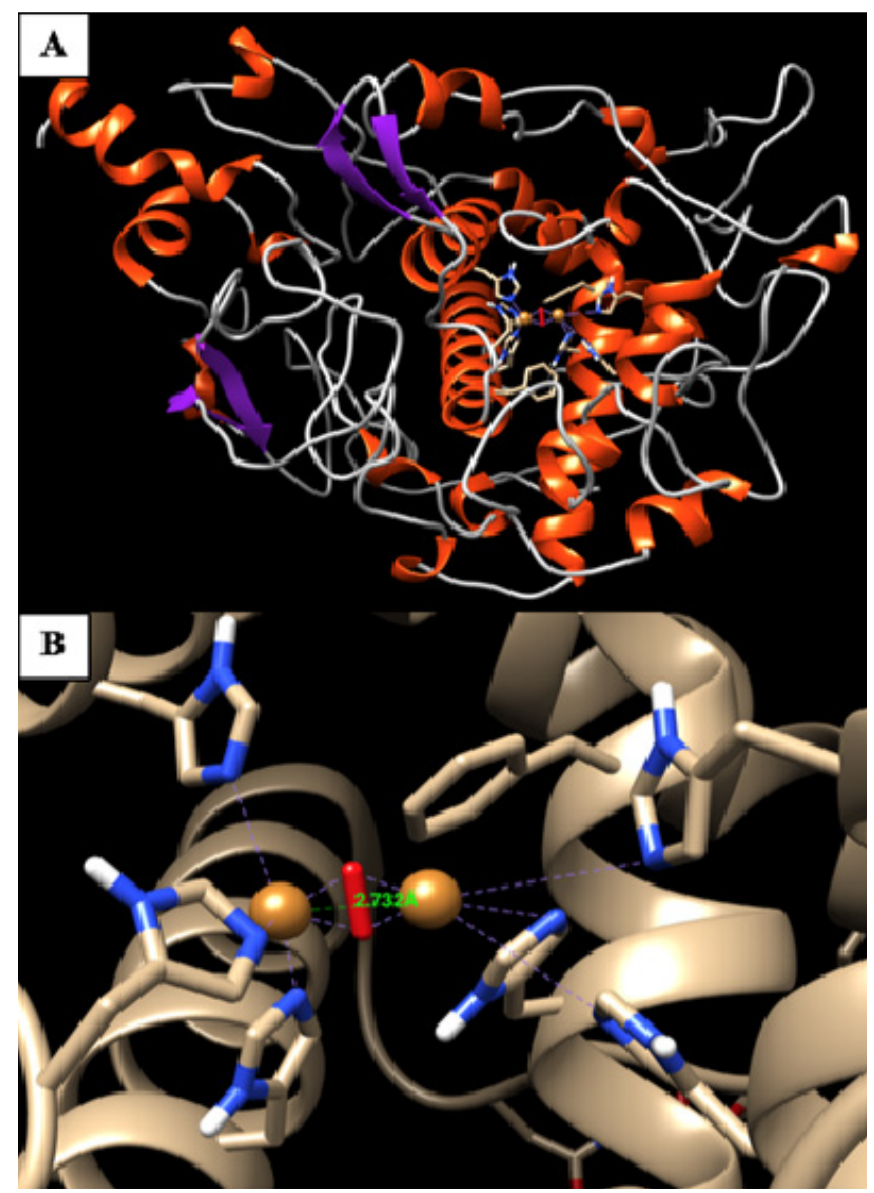

Figure I (A) Tyr homology model colored by secondary structure. $\alpha$-Helices (and copper atoms) are highlighted in orange, beta sheets are highlighted in purple, coils are highlighted in gray. (B) The ribbon structure of the Tyr active site with coordinating copper His residues displayed. The distance between the copper atoms is $2.7-2.8 \AA$, and the $\mathrm{O}_{2}$ molecule is located between them, stabilizing the two atoms.

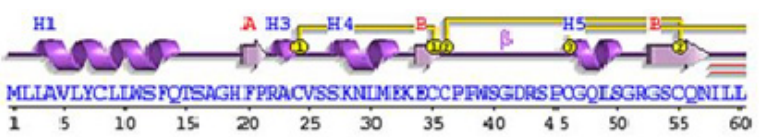

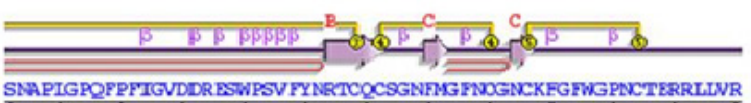

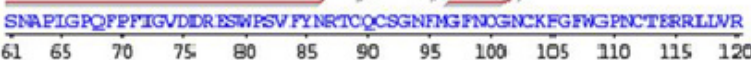
H6 $\mathrm{H7} \quad$ H8 $\mathrm{A} \quad$ H9

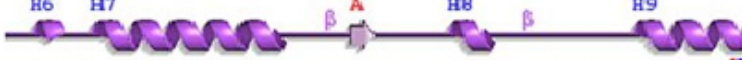
RNIFDISA.PEXDKFFAYITIAKHTISSDYVI PIGIXGGMKNGSTEMENDINIYDLFWMG

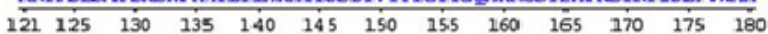
Par

YYVSMDALIOGSETWRDIDFÄHEAPA FLFWHRLFLLRWDDEIOKLIGDENFTIPYWLNRD

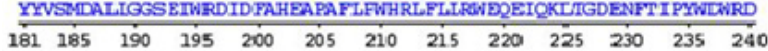

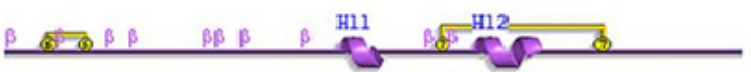
AEKCDICTDEN-10GQHPINPNLISPAS FFSSWQIVCSRLEEYNSHQSICNGT PEGPLRRN

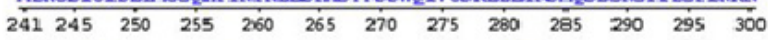
FGNFIKSRTPRLPSSADVEFCISIMQY ESGSMDKAANISFRENLLEGFASPLIGIADASCS

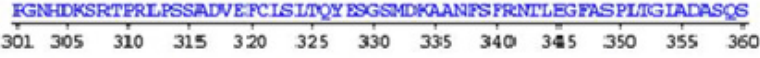

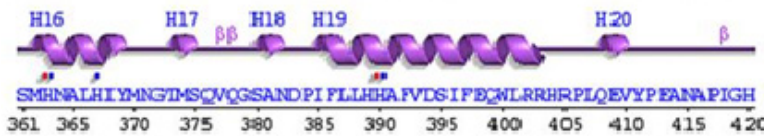

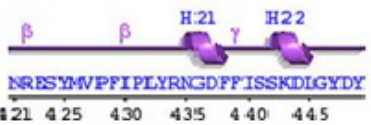

Figure 2 Sequence of the intra-melanosomal domain of human Tyr with respective secondary structure elements labeled. Obtained using the pdbsum generate server (https://www.ebi.ac.uk/thornton-srv/databases/pdbsum/ Generate.html).
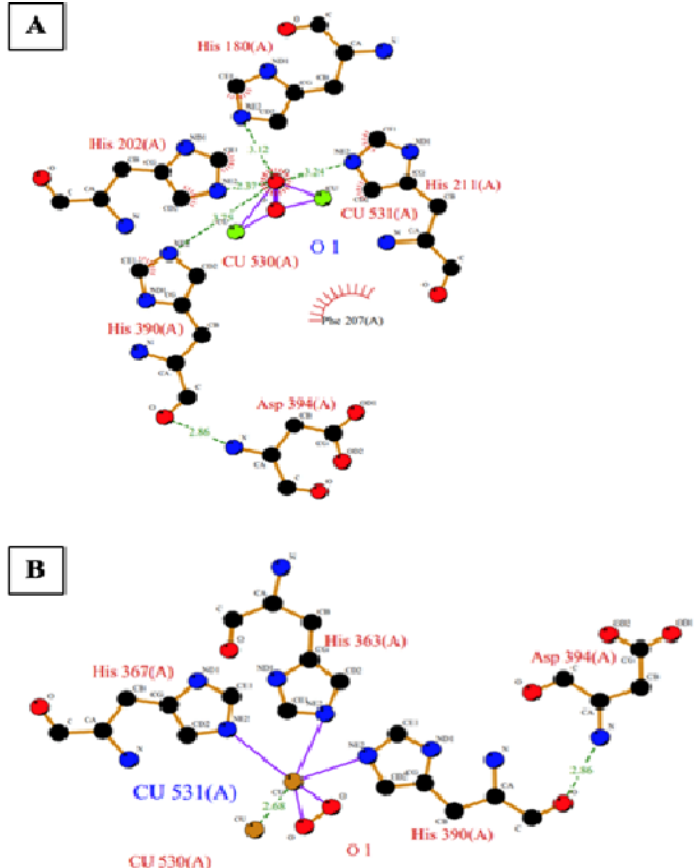

Figure 3 Interactions within the active site. A. Histidine interactions with CuA. B. Histidine interactions with CuB. Obtained using the pdbsum generate server (https://www.ebi.ac.uk/thornton-srv/databases/pdbsum/Generate. 


\section{Structural changes caused by OCAIB mutations}

In order to define key characteristics of OCA1 and molecular differences between OCA1A and OCA1B, eight OCA1B mutants and eleven active site His OCA1A mutants were analyzed. The $\Delta \Delta \mathrm{G}$ value and unfolding parameter were calculated for each OCA1B mutant using UMS (Table 1).

Foldability parameters were listed for each residue of interest, highlighting each residue's importance to structural and thermodynamic stability (Table 2).

Table I OCAIB mutant simulation results

\begin{tabular}{lllll}
\hline Mutation & $\Delta \Delta \mathbf{G}^{*}$ & Unfolding & Predicted Effect & $\Delta \Delta \mathbf{V}$ \\
\hline W39R & 1.2 & 0.88 & Severe & 1248 \\
R77G & 5.7 & 1 & Severe & 1670 \\
KI42M & -1.7 & 0.06 & Severe & 928 \\
S323R & 0.8 & 0.8 & Moderate & 255 \\
T325A & -0.3 & 0.39 & Severe & 540 \\
M370V & 4.5 & 1 & Severe & 579 \\
V393D & 4.8 & 1 & Severe & 1656 \\
R402G & 2.1 & 0.97 & Severe & 685 \\
\hline
\end{tabular}

*For mutants with $\Delta \Delta \mathrm{G}$ values below 0 , we assume the structures to have a more stable but still non-native structure. Therefore, they are labeled as severe

Table 2 Foldability parameters for OCAIB mutations

\begin{tabular}{lllllllll}
\hline Residue & W39 & R77 & K142 & S323 & T325 & M370 & V393 & R402 \\
\hline Foldability & 1.83 & 18.95 & 8.79 & 5.75 & 0 & 15.96 & 14.83 & 12.38 \\
\hline
\end{tabular}

For these residues, R77, M370, V393, and R402 were predicted to have the greatest destabilizing effect with $\Delta \Delta \mathrm{G}=2.1-5.7 \mathrm{kcal} /$ mol. K142 is in $\alpha$-helix H7, located away from the active site. S323 and T325 are residues in the coil between $\alpha$-helix H14 and $\alpha$-helix $\mathrm{H} 15$, located on the periphery of the protein, and do not contribute to the secondary/tertiary structure of Tyr. R77 is located within the beta hairpin of beta sheet B. M370 is located one residue after transmembrane $\alpha$-helix H16. V393 and R402 are both located within transmembrane $\alpha$-helix H19. W39 is located within the EGF-like motif (Figure 2).

Mutant structures were overlaid onto the Tyr structure at $100 \mathrm{~ns}$ to identify visible structural changes (Figure 4). No visible differences were found within the transmembrane $\alpha$-helices, active site, EGF-like domain, or glycan-rich regions. The $\Delta \Delta \mathrm{G}$ value of unfolding did not correlate with differences in secondary or tertiary structure in OCA1B mutants. K142M, a stable yet non-native mutant, and R77G, the most unstable mutant, exhibited similar minor differences in random loop fluctuations, but no difference in Tyr regions of importance (EGFlike domain, active site, and secondary structure). The structure comparison of Tyr and K142M yielded an RMSD across pruned atom pairs of $1.2 \AA$ and an RMSD across all atom pairs of $2.4 \AA$. The structure comparison of Tyr and R77G yielded an RMSD across pruned atom pairs of $1.3 \AA$ and an RMSD across all atom pairs of 3.3 $\AA$.

Solvent-accessible volume and cavity volume within the protein were collected for each OCA1B mutant variant in MD simulations in order to identify a link between these parameters and Gibbs free energy changes. Solvent-accessible volume was calculated by YASARA. Calculated by Mole 2.5, total cavity volume was determined as the sum of the three largest cavities within the protein. Over $100 \mathrm{~ns}$, the difference in solvent-accessible volume increased directly for each mutant with the $\Delta \Delta \mathrm{G}$ value (Table 1 ). The $\Delta \Delta \mathrm{SA}$ value compared to $\Delta \Delta G$ had a Pearson's Coefficient of 0.55 , indicating a low association between the severity of the mutation as described by $\Delta \Delta \mathrm{G}$ and the total surface of the protein (Figure 3B). The $\Delta \Delta \mathrm{V}$ value compared to $\Delta \Delta \mathrm{G}$ had a Pearson's Coefficient of 0.63 , indicating that the total volume of the protein increases with the severity of the mutation (Figure 5C).

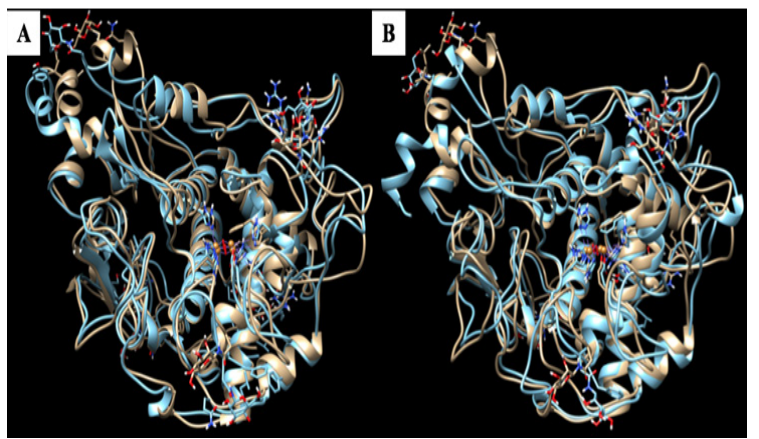

Figure 4 The superposition of Tyr native structure and KI42M and R77G mutant variants obtained after $100 \mathrm{~ns}$ MD run. (A) The superposition of the protein atomic structures of native Tyr (bronze) and the KI42M mutant variant are overlaid onto one another using MD at 100 ns. Both proteins are represented by ribbon structures. There were no visible differences found in regions of importance. The RMSD across pruned atom pairs was I.2 $\AA$. (B) The ribbon structures of Tyr (bronze) and R77G (cyan) are overlaid onto one another at $100 \mathrm{~ns}$. There were no visible differences found in regions of importance. The RMSD across pruned atom pairs was $1.3 \AA$.

While OCA1A is characterized as a complete loss of Tyr function, OCA1B exhibits reduced enzymatic activity. Tyr seems to complete the folding process; however, mutations within the protein result in a partial loss of activity. According to our data, once OCA1B mutants 
had completed the folding process, the protein structure began to slowly expand, creating cavities that channeled water molecules to the interior of the protein. The most severe mutations of Tyr resulted in large cavities of up to $6500 \AA^{3}$ of a difference compared to the native Tyr (Figure 5C). Cavities were then analyzed for location commonality in regard to secondary structures, the EGF-like domain, and the active site.
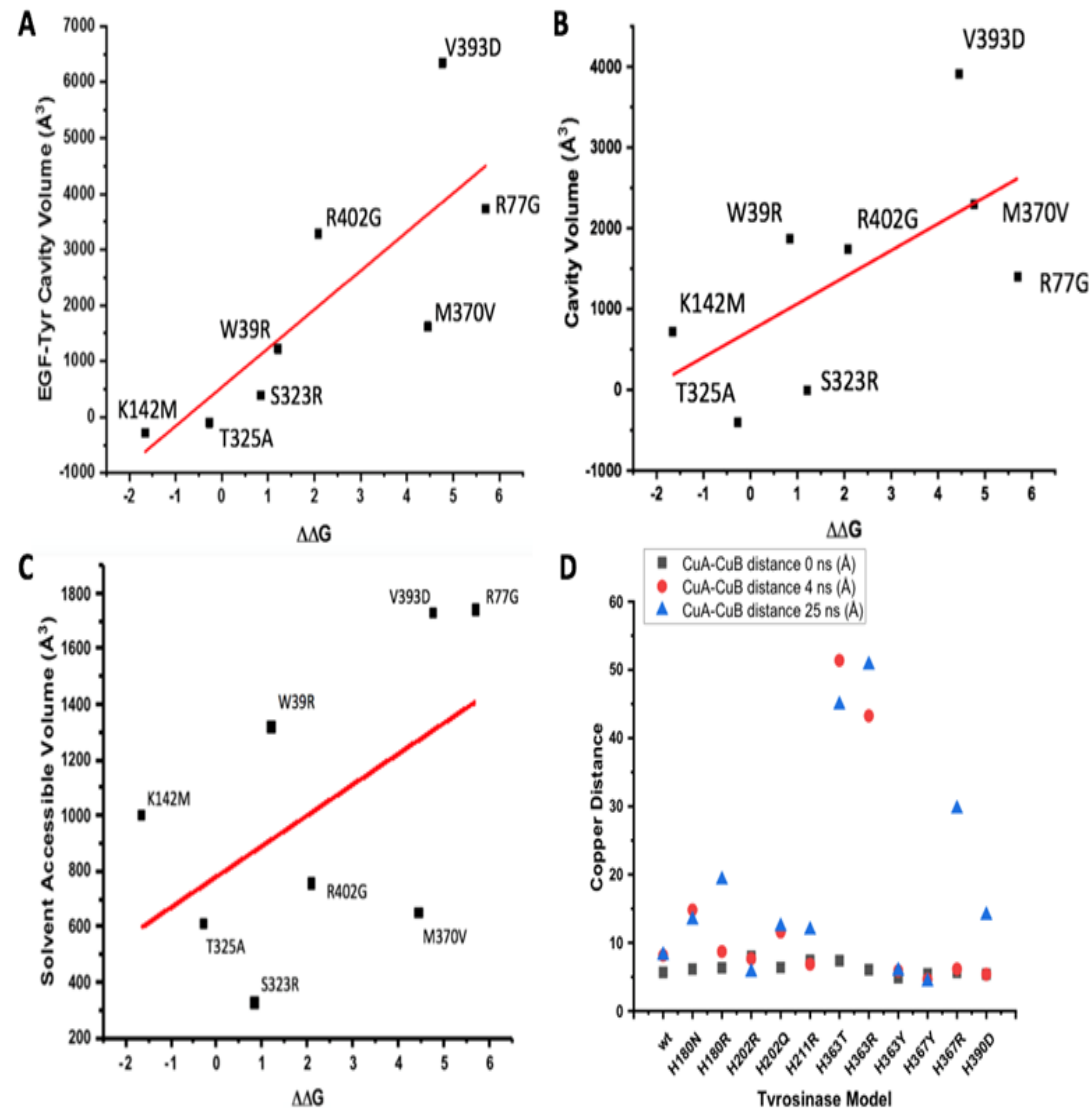

Figure 5 Changes in cavity volume, solvent-accessible area and volume, and inter-atomic copper distances for OCAI mutations. (A) Graph of $\Delta \Delta \mathrm{G}$ vs change in EGF-Tyr interface cavity volume from difference at 0 ns and $100 \mathrm{~ns}\left(\Delta \Delta \mathrm{V}_{\mathrm{cav}}\right)$ for OCAIB mutations. Pearson's coefficient of 0.80 . Adjusted R-square value of 0.57. Regression equation: $\Delta \Delta V_{\text {cav }}=749.1 \times \Delta \Delta G+423.0$. (B) Graph of $\Delta \Delta G$ vs change in solvent-accessible volume from difference at $0 \mathrm{~ns}$ and $100 \mathrm{~ns}$ for OCAIB mutations. Pearson's coefficient of 0.55 . Adjusted R-square value of 0.18 . (C) Graph of $\Delta \Delta \mathrm{G}$ vs change in cavity volume from difference at 0 ns and $100 \mathrm{~ns}$ for OCAIB mutations. Pearson's coefficient of 0.63 . Adjusted R-square value of 0.30 . (D) Copper distances measured at $0 \mathrm{~ns}, 4 \mathrm{~ns}$, and $25 \mathrm{~ns}$ for Tyr and each His active site mutation. For mutants with copper distances $<10 \AA$ at $25 \mathrm{~ns}$, it is important to note that the copper initially coordinated by two His residues had lost all coordination at $25 \mathrm{~ns}$.

Large Tyr cavities were found near the EGF-like domain, which is located within the first 100 residues of the protein. The EGF-like domain is a cysteine-rich region that contains five disulfide bridges (Figure 6). ${ }^{21}$ The hydrophobic cavity between the EGF-like domain and the rest of the Tyr domain (EGF-Tyr interface) contains many large aromatic residues (F98, F105, F176, W210, F429, Y433, and F438). This interface was monitored to assess destabilization in Tyr mutants in OCA1B (Table 3). The EGF-Tyr interface volume was 422 $\AA^{3}$ at 100 ns for native Tyr. Mutants with $\Delta \Delta \mathrm{G}<0$ contained cavities with decreased size compared to Tyr within the cysteine-rich domain.
Mutants with $\Delta \Delta \mathrm{G}>0$ contained significantly larger cavities within the EGF-Tyr interface, leading to destabilization of the protein (Figure 5A). A strong correlation was found between the two parameters. Cavities within or near the active site show no correlation with $\Delta \Delta \mathrm{G}$ values and, therefore, are not listed.

Large cavities within the EGF-Tyr interface suggest it to be a source for destabilization in OCA1B mutants. At $100 \mathrm{~ns}$, the cavity encompassing the EGF-Tyr interface had perforated throughout the protein, ultimately affecting the active site in severe mutations. The native Tyr and weak mutants did not display these large cavities.

Table 3 Size of cavity between EGF-like domain and Tyr domain for each mutant

\begin{tabular}{llllllllll}
\hline Mutant & Tyr & KI42M & T325A & S323R & W39R & R402G & M370V & V393D & R77G \\
\hline Cavity $\left(\AA^{3}\right)$ & 422 & 137 & 320 & 810 & 1648 & 3709 & 2041 & 6766 & 4154 \\
\hline
\end{tabular}




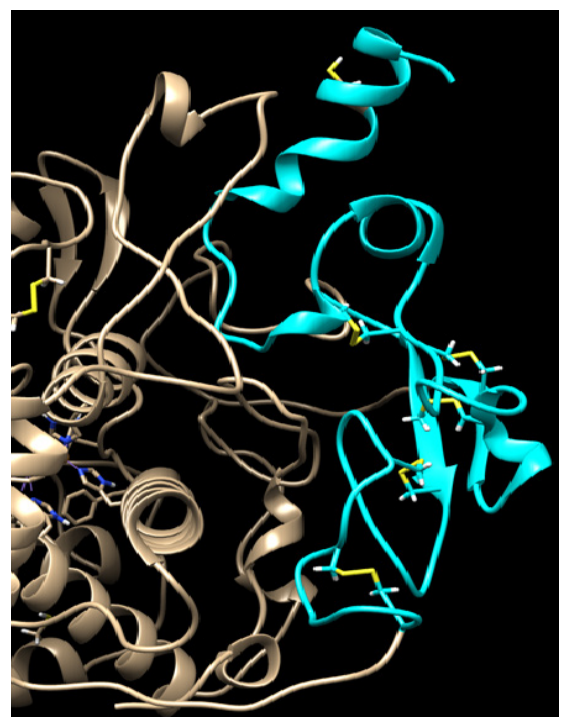

Figure 6 The cavity localized between tyrosinase and EGF-like domains. The EGF-like domain is highlighted in blue and the rest of Tyr is colored bronze. The disulfide bridges are displayed.

\section{Human tyrosinase active site}

Within the active site of tyrosinase there is a bundle of four alpha helices that contain six copper coordinating histidine residues. The two copper atoms are stabilized and tightly held together (2.7-2.9 $)$ by two oxygen atoms (Figure 1B). Mutations in copper coordinating histidine residues could cause a partial or complete loss of protein activity. ${ }^{4}$ In order to find the mechanism leading to protein instability, we analyzed albinism causing genetic mutations localized in histidines coordinating copper within the active site of Tyr. The foldability parameter for each active site histidine residues is presented in Table 4. For these residues, mutations to $\mathrm{H} 202$ and $\mathrm{H} 211$ were predicted to have the greatest destabilizing effect. UMS calculations for individual mutations predicted significant changes causing a loss of protein stability or a stabilizing effect leading to a non-native stable structure (Table 5).

Table 4 Foldability parameters for active site mutations

\begin{tabular}{lllllll}
\hline Residue & $\mathbf{H} 180$ & $\mathbf{H 2 0 2}$ & $\mathrm{H} 2 \mathrm{II}$ & $\mathbf{H 3 6 3}$ & $\mathbf{H 3 6 7}$ & $\mathrm{H} 390$ \\
\hline Foldability & 3.97 & 14.83 & 14.93 & 3.91 & 0.99 & 3.99 \\
\hline
\end{tabular}

Table 5 OCAIA mutation simulation results containing oxygen binding copper

\begin{tabular}{lllll}
\hline Mutation & $\Delta \Delta \mathbf{G}^{*}$ & Unfolding Parameter & $\Delta \Delta \mathbf{V}$ & $\Delta \Delta \mathbf{S A}$ \\
\hline HI80N & -0.95 & 0.17 & 563 & 705 \\
HI80R & 2.16 & 0.97 & 1178 & 1020 \\
H202R & 2.81 & 0.99 & 66 & 49 \\
H202Q & 1.36 & 0.91 & -294 & 54 \\
H2IIR & 9.24 & 1.00 & -435 & -376 \\
H363T & -1.46 & 0.08 & 1255 & 1419 \\
H363R & -0.80 & 0.21 & 1505 & 1610 \\
H363Y & 1.58 & 0.93 & -632 & -314 \\
H367Y & 0.93 & 0.82 & -285 & -36 \\
H367R & -0.91 & 0.18 & 813 & 927 \\
H390D & -0.17 & 0.43 & -178 & 146 \\
\hline
\end{tabular}

*For mutants with $\Delta \Delta \mathrm{G}$ values below 0 , we assume the structures to have a more stable but still non-native structure. Therefore, they are labeled as severe

The effect of mutations was simulated using MD. Each mutant variant was generated as described in the Methods section. After simulation of each OCA1A mutant was completed, the differences of the molecular-accessible volume and surface area between $0 \mathrm{~ns}$ and $100 \mathrm{~ns}$ were recorded in order to assess structural changes (Table 5). No correlation was found between mutation severity and $\Delta \Delta \mathrm{V}$ (Pearson's Coefficient $=-0.51$ ) or $\Delta \Delta \mathrm{SA}$ (Pearson's Coefficient $=$ -0.62 ), suggesting the correlation between $\Delta \Delta \mathrm{G}$ and OCA1B mutant expansion might not apply to active site His mutants. No significant visual differences were found between Tyr and the OCA1A mutants, and there was no correlation with the $\Delta \Delta \mathrm{G}$ values (Figure 7). The structure comparison of Tyr and H363T, the most stable OCA1A mutant, produced an RMSD across pruned atom pairs of $1.1 \AA$ and an RMSD across all atom pairs of $3.1 \AA$ at 100 ns. The structure comparison of Tyr and H211T, the most severe OCA1A mutant, produced an RMSD across pruned atom pairs of $1.2 \AA$ and an RMSD across all atom pairs of $3.1 \AA$ at $100 \mathrm{~ns}$.

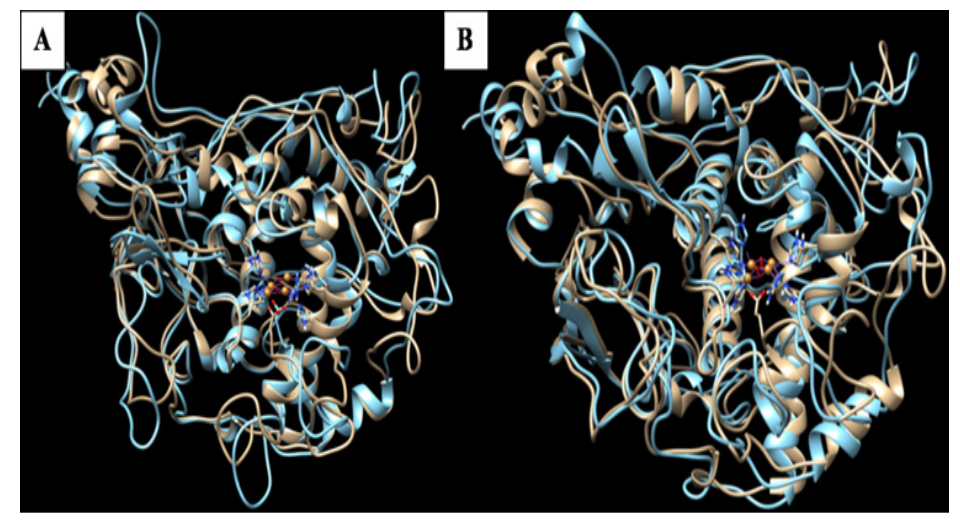

Figure 7 The superposition of Tyr native structure and H363T and H2I IR mutant variants were obtained after 100 ns MD run. (A) The ribbon structures of Tyr (bronze) and H363T (cyan) are overlaid onto one another at $100 \mathrm{~ns}$. The RMSD across pruned atom pairs is I.I A.. (B) The ribbon structures of Tyr (bronze) and H2I IR (cyan) are overlaid onto one another at I00 ns. The RMSD across pruned atom pairs is I.2 $\AA$. 


\section{Effect of $\mathrm{O}_{2}$ in histidine active site mutant function in OCAIA}

The glycosylated Tyr and all eight OCA1B mutant simulations were run with and without $\mathrm{O}_{2}$-binding copper within the active site to define its role in Tyr function. After $25 \mathrm{~ns}$, the copper distance for Tyr expanded to $8.2 \AA$. None of the mutants were located within the active site or were able to affect copper stability, so there was no major difference with the OCA1B mutants. Although the stabilizing effect of $\mathrm{O}_{2}$ was removed, three His residues still coordinated each copper and held them within the active site. The dioxygen molecule bound to both coppers, thought to be integral to protein stability, was removed from Tyr and the active site mutations. Simulations for all

Table 6 OCAIA mutation simulation results without oxygen binding copper models without dioxygen were run for $25 \mathrm{~ns}$, and the copper distance was measured at $0 \mathrm{~ns}, 4 \mathrm{~ns}$, and $25 \mathrm{~ns}$ (Table 6). For Tyr without $\mathrm{O}_{2}$, the copper atoms separated to $8.2 \AA$ at $4 \mathrm{~ns}$ and were held stable for the rest of the simulation. For each active site His mutant, one of the two coppers lost coordination. The copper coordinated by two His residues was no longer held in place in the active site, and it either moved out and away from the two His residues or out of the active site entirely, indicating that the absence of $\mathrm{O}_{2}$ leads to protein instability in active site His mutations (Figures 5D \& 8). In those mutants, the other copper was coordinated by three His residues and did not exhibit any signs of loss of coordination, keeping its relative position throughout the simulation. These results suggest that OCA1A mutant misfolding could be associated with protein instability within the active site.

\begin{tabular}{llll}
\hline Tyrosinase Model & Copper distance $\mathbf{0}$ ns $(\mathbf{A})$ & Copper distance $\mathbf{4}$ ns $(\mathbf{A})$ & Copper distance $\mathbf{2 5}$ ns $(\mathbf{A})$ \\
\hline Tyrosinase Wild Type & 5.7 & 8.2 & 8.2 \\
HI80N & 6.1 & 14.8 & 13.4 \\
HI80R & 6.3 & 8.7 & 19.2 \\
H202R & 8.0 & 7.7 & 5.7 \\
H202Q & 6.4 & 11.6 & 12.4 \\
H2IIR & 7.4 & 6.8 & 11.9 \\
H363T & 7.4 & 51.4 & 44.9 \\
H363R & 6.1 & 43.3 & 50.8 \\
H363Y & 4.9 & 5.9 & 5.9 \\
H367Y & 5.4 & 4.6 & 4.3 \\
H367R & 5.7 & 6.2 & 29.6 \\
H390D & 5.4 & 5.4 & 14.0 \\
\hline
\end{tabular}

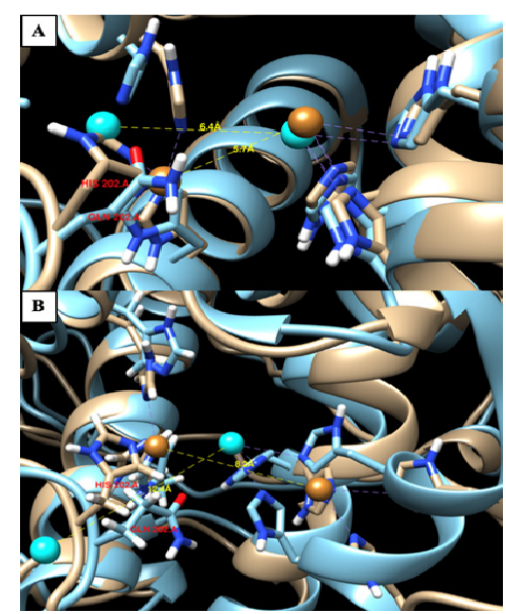

Figure 8 The superposition of Tyr without $\mathrm{O}_{2}$ and $\mathrm{H} 202 \mathrm{Q}$ without $\mathrm{O}_{2}$. (A) The ribbon structures of the Tyr (bronze) and the H202Q mutant (cyan) are overlaid onto one another at 0 ns. Histidine residues required for copper coordination are shown in stick form with copper atoms in bronze for the Tyr and cyan for the $\mathrm{H} 202 \mathrm{Q}$ mutant. The distance between the two copper atoms is $5.7 \AA$ for the WT and $6.4 \AA$ for the mutant. (B) The ribbon structures of the Tyr (bronze) and the H202Q mutant (cyan) are overlaid onto one another at 25 ns. Histidine residues required for copper coordination are shown in stick form with copper atoms in bronze for Tyr and cyan for the $\mathrm{H} 202 \mathrm{Q}$ mutant. The distance between the two coppers is $8.2 \AA$ for Tyr and $12.4 \AA$ for the mutant. Q202 is not able to coordinate the copper ion, increasing the separation between the atoms. The active site of $\mathrm{H} 202 \mathrm{Q}$ is larger than the WT, due to a slight unfolding of the protein.

\section{Discussion}

Oculocutaneous albinism type 1 (OCA1) is an autosomal recessive disorder caused by mutations in the tyrosinase gene. OCA1 exists in two forms: OCA1A and OCA1B. OCA1A is caused by a full loss of the human tyrosinase protein (Tyr), leading to an absence of pigment in skin, hair, and eyes, while OCA1B has reduced Tyr catalytic activity and pigment. In this work, Tyr and mutant variants were built through homology modeling, glycosylated in silico, minimized, and simulated using $100 \mathrm{~ns}$ molecular dynamics in water. For OCA1B mutants, cavity size is linked to $\Delta \Delta \mathrm{G}$ values for mutants, suggesting that partial loss of Tyr in OCA1B is associated with the destabilizing effect of EGF-like domain movement. In OCA1A, active site mutation simulations indicate that the absence of $\mathrm{O}_{2}$ leads to protein instability. OCA1B mutants are described in severity by the size of the cavity within the EGF-Tyr interface, while active site OCA1A mutants are unable to fully coordinate copper, leading to an absence of $\mathrm{O}_{2}$ and Tyr instability. In patients with known genotypes, $\Delta \Delta \mathrm{G}$ values may help identify the severity of OCA1 by assessing either the allosteric effect of the EGF-Tyr cavity in OCA1B or the active site instability in OCA1A.

Many conserved regions exist in the tyrosinase family proteins, one of which is an EGF-like domain. ${ }^{21}$ This motif is comprised of a cysteine-rich region that contains five disulfide bridges, assisting with protein stability. Although the majority of OCA1B mutations are not located within the EGF-like motif, they seem to have an allosteric affect that slowly decreases Tyr function. When $\Delta \Delta G<0$, the cavity between the EGF-like domain and the Tyr domain decreases compared 
to Tyr. The mutant cavity size increases directly with $\Delta \Delta \mathrm{G}$ values, as the EGF-like domain moves away from the Tyr domain. Due to the hydrophobic character of the cavity, the continuous expansion and channeling of water is thermodynamically unfavorable and is accompanied by a negative enthalpy and entropy change. This leads to destabilization of the Tyr-EGF interface that propagates throughout the entire domain, resulting in an unstable protein. This gradual process can contribute to a decrease in Tyr activity and OCA1B. Simulation results indicate that the mutants only partially denature, meaning they still might conserve some functionality. Active site histidine mutations show no correlation with EGF-like domain movement and do not display signs of unfolding.

The effect of cavity formation due to mutation changes, in part, could be interpreted at the level of interatomic interactions. Indeed, mutation K142M results in the removal of two hydrogen bonds. The first is between hydrogen $1 \mathrm{HZ}$ and oxygen OD2 atoms of K142$1 \mathrm{HZ}$ and D174-OD2, and is $2.0 \AA$. The second is between K142$2 \mathrm{HZ}$ and D174-OD1, and is $2.7 \AA$. These bonds are located on the surface of the protein and, in the K142M mutation, M142 rotates towards the interior. W39 is located between R52 and R43, and the W39R mutation can result in repulsive interactions between three consecutive arginine residues. W39R is $4.1 \AA$ away from R52 and 4.4 $\AA$ away from R43, leading to greater destabilization in the EGF-like region and a greater $\Delta \mathrm{G}$ value. The effect of the M370Vmutation is less definitive. Arginine R77 forms two hydrogen bonds with aspartic acid D75. The first is between R77-1HH1 and D75-OD1, and its length is $1.7 \AA$. The second is between R77-HE and D75-OD2, and is $1.8 \AA$. The $\mathrm{R} 77 \mathrm{G}$ mutation removes both hydrogen bondsand replaces Arg with a glycine residue. S323R and T325A do not experience much of a change in environment. R402 is located on the surface of the protein at the end of $\alpha$-helix H19. The R402G mutation may affect the stability of the $\alpha$-helix, though it is not shown in the simulation. V393D introduces a polar residue within the hydrophobic core and forms the hydrophobic bond D393:HD1-H389:OD1 of length 1.7 $\AA$. The simulation does not show a change in the shape of $\alpha$-helix H19; however, the D393 changes orientation from V393, which could destabilize an $\alpha$-helix containing His-coordinating copper. While mutations might have an allosteric effect on the EGF-Tyr interface, large $\Delta \Delta \mathrm{G}$ values can also be attributed to changes within those mutations' environments for most cases.

There are six total His-Cu coordinating bonds in Tyr. When a His residue is mutated within the active site, five other His-Cu coordinating bonds are still able to keep the protein intact. After $100 \mathrm{~ns}$ of His active site OCA1A mutant simulations, there was no evidence of denaturation or significant structural differences between the mutants and Tyr. This suggests that His active site mutations are destabilized prior to protein folding, since OCA1A mutants are known to be denatured. Parameters derived from the UMS calculation indicate that OCA1A active site histidine residues are not necessarily critical for protein folding; however, this parameter does not account for the loss of copper coordination (Table 4). Lack of significant change in free energy suggests that active site mutations are not able to coordinate the copper before the protein folding process is completed.

The OCA1A mutant folding process may hinge on oxygen-binding copper bringing together two halves of the protein, which could build the protein catalytic site by the formation of native 4-helix bundle motif. To test protein structure stability without the oxygen, the $\mathrm{O}_{2}$ molecule was removed from the protein and simulated for 100 ns. Without the oxygen molecules the copper atoms are no longer held tightly together, and their separation gradually increases, thereby affecting the substrate binding site located between the $\alpha$-helices and destabilizing the protein structure. For almost every mutant, the solvent-accessible surface area and solvent-accessible volume increased compared to the Tyr structure. Additionally, when the histidine residues are no longer completely able to coordinate copper with the help of dioxygen, the surface area and volume of the protein accessible to solvent begins to increase greatly in comparison to the surface area and volume of the tyrosine wild type (Tables 5, 6).

In patients with known OCA1 genotypes, computer simulations performed in our study may help identify the severity of OCA1. By assessing either the allosteric effect of the EGF-Tyr cavity in OCA1B or the active site instability in OCA1A, we can guide treatment tailored to individual patients with OCA1. In disease, this can help identify the Tyr molecular mechanism associated with partial or complete protein misfolding.

\section{Conclusions}

From our analysis of human tyrosinase structure, we demonstrated an association between changes in mutant variants and OCA1 phenotypes. Indeed, OCA1B mutants are described in severity by the size of the cavity within the EGF-Tyr interface, while active site OCA1A mutants are unable to fully coordinate copper, leading to an absence of $\mathrm{O}_{2}$ and Tyr instability. In patients with known genotypes, free energy changes may help identify the severity of the disease by assessing either the allosteric effect of the EGF-Tyr cavity in OCA1B or the active site instability in OCA1A.

\section{Acknowledgments}

The authors thank present and former members of our group and the NEI ITMB team Drs. Andrei Volkov and Mikhail Laryukhin for their help and discussion input during the completion of this study. Also, the authors thank Cindy Clark, NIH Library Writing Center, for manuscript editing assistance.

\section{Conflicts of interest statement}

None declared.

\section{Funding}

This research was supported by the Intramural Research Program of the NIH, National Eye Institute (ZIA EY000476-10 to Y.V.S).

\section{References}

1. Olivares C, Solano F. New insights into the active site structure and catalytic mechanism of tyrosinase and its related proteins. Pigment Cell Melanoma Res. 2009;22(6):750-760.

2. Garcia-Borron JC, Solano F. Molecular anatomy of tyrosinase and its related proteins: beyond the histidine-bound metal catalytic center. Pigment Cell Res. 2002;15(3):162-173.

3. Gronskov K, Ek J, Brondum-Nielsen K. Oculocutaneous albinism. Orphanet J Rare Dis. 2007;2(2):43.

4. Dolinska MB, Kus NJ, Farney SK, et al. Oculocutaneous albinism type 1: link between mutations, tyrosinase conformational stability, and enzymatic activity. Pigment Cell Melanoma Res. 2017;30(1):41-52.

5. Lai X, Soler-Lopez M, Wichers HJ, et al. Large-Scale Recombinant Expression and Purification of Human Tyrosinase Suitable for Structural Studies. PLoS One. 2016;11:e0161697. 
6. Lai X, Wichers HJ, Soler-Lopez M, et al. Structure of Human Tyrosinase Related Protein 1 Reveals a Binuclear Zinc Active Site Important for Melanogenesis. Angew Chem Int Ed Engl. 2017;56(33):9812-9815.

7. Lai X, Wichers HJ, Soler-Lopez M, et al. Structure and Function of Human Tyrosinase and Tyrosinase-Related Proteins. Chemistry. 2018;24(1):47-55.

8. Branza-Nichita N, Petrescu AJ, Negroiu G, et al. N-glycosylation processing and glycoprotein folding-lessons from the tyrosinase-related proteins. Chem Rev. 2000;100:4697-4712.

9. Branza-Nichita N, Negroiu G, Petrescu AJ, et al. Mutations at critical $\mathrm{N}$-glycosylation sites reduce tyrosinase activity by altering folding and quality control. J Biol Chem. 2000;275:8169-8175.

10. Snell RS, Lemp MA. Clinical anatomy of the eye. USA: Blackwel Science, Malden, MA; 1988.

11. McCafferty CL, Sergeev YV. In silico Mapping of Protein Unfolding Mutations for Inherited Disease. Sci Rep. 2016;6:37298.

12. McCafferty CL, Sergeev YV. Dataset of eye disease-related proteins analyzed using the unfolding mutation screen. Sci Data. 2016;3: 160112.

13. Farney SK, Dolinska MB, Sergeev YV. Dynamic analysis of human tyrosinase intra-melanosomal domain and mutant variants to further understand oculocutaneous albinism type 1. J Anal Pharm Res. 2018;7:621-632.
14. Dolinska MB, Kovaleva E, Backlund P, et al. Albinism-causing mutations in recombinant human tyrosinase alter intrinsic enzymatic activity. PLoS One. 2014;9:e84494.

15. Pettersen EF, Goddard TD, Huang CC, et al. UCSF Chimera-a visualization system for exploratory research and analysis. J Comput Chem. 2004;25:1605-1612.

16. Dolinska MB, Wingfield PT, Young KL, et al. The TYRP1-mediated protection of human tyrosinase activity does not involve stable interactions of tyrosinase domains. Pigment Cell Melanoma Res. 2019;32(6):753-765.

17. Kim JH, Johannes L, Goud B, et al. Noninvasive measurement of the $\mathrm{pH}$ of the endoplasmic reticulum at rest and during calcium release. Proc Natl Acad Sci U S A. 1998;95:2997-3002.

18. Bellono NW, Escobar IE, Oancea E. A melanosomal two-pore sodium channel regulates pigmentation. Sci Rep. 2016;6:26570.

19. Sehnal D, Svobodova Varekova R, Berka K, et al. MOLE 2.0: advanced approach for analysis of biomacromolecular channels. J Cheminform. 2013;5:39.

20. Schymkowitz J, Borg J, Stricher F, et al. The FoldX web server: an online force field. Nucleic Acids Res. 2005;33:W382-388.

21. Jackson IJ, Chambers DM, Tsukamoto K, et al. A second tyrosinaserelated protein, TRP-2, maps to and is mutated at the mouse slaty locus. EMBO J. 1992;11(2):527-535. 